\title{
latrogenic lipoid pneumonia in an adult horse
}

Lucy Metcalfe*, Carolyn Cummins, Eva Maischberger, Lisa Katz

Section of Veterinary Clinical Studies, University Veterinary Hospital, School of Agriculture, Food Science and Veterinary Medicine, University College Dublin, Belfield, Dublin 4, Ireland

Lucy Metcalfe, University Veterinary Hospital, School of Agriculture, Food science and Veterinary Medicine, University College Dublin, Dublin 4, Ireland

lucy.metcalfe@ucd.ie

\begin{abstract}
A 20-year-old gelding presented with a history of acute respiratory distress which began immediately after administration of a mineral oil and water mix, via nasogastric intubation, for treatment of suspected gastrointestinal dysfunction. An initial presumptive diagnosis of acute lipoid pneumonia was made; this was further supported by evidence of arterial hypoxaemia and oxygen desaturation on arterial blood gas analysis, ultrasonographic signs of bilateral ventral lung consolidation and a mixed bronchoalveolar-interstitial lung pattern seen on thoracic radiographs. Despite intensive supportive therapy the horse's condition continued to deteriorate and the decision was made for humane euthanasia. Gross necropsy findings supported the clinical diagnosis of lipoid pneumonia.
\end{abstract}

KEYWORDS: Lipoid pneumonia, mineral oil, nasogastric intubation

Irish Veterinary Journal

Volume 63 Number 52010

\section{INTRODUCTION}

Mineral oil (liquid paraffin) is commonly used in equine practice as a lubricant in cases of gastrointestinal impaction and as a purgative in cases of potential toxicosis (Davis et al., 2001). However, administration of mineral oil to horses via nasogastric intubation does carry a minor risk of causing mineral oil-induced pneumonitis (lipoid pneumonia). Lipoid pneumonia is a severe inflammatory reaction to the presence of oil in the lungs, which is usually fatal when it occurs in horses.. The guarded prognosis is due in part to the fact that mineral oils appear to alter the viscoelastic properties of secretions as well as the mucociliary transport mechanism (Lauque et al., 1990, Scarratt et al., 1998). Human studies have shown that the clearance of aspirated oil from the respiratory tract is difficult with oily substances typically emulsified by lung lipase and engulfed by alveolar macrophages thus triggering local inflammation (Franquet et al., 1997, Sias et al., 2009). Lopez (2001) reports that proinflammatory mediators open endothelial gaps, increasing the permeability of blood vessels and resulting in leakage of plasma and fibrinogen into the alveoli which progressively obliterates airspaces, causing consolidation of the lung. Due to the infrequency of the problem, there are few published reports describing the clinical findings associated with pulmonary aspiration of mineral oil in the horse. Scarratt et al. (1998) and Davis et al. (2001) reported the clinical presentation to be either acute or chronic in nature, depending upon the quantity of mineral oil aspirated. In acute cases, respiratory distress rapidly develops, whereas chronic cases may develop a cough, abnormal lung sounds and intermittent fever over several weeks, finally presenting at an advanced stage (Davis et al., 2001). The following report describes a case of acute lipoid pneumonia in a horse that was being treated for gastrointestinal dysfunction with the risk factors, clinical presentations and diagnostic options discussed.

\section{HISTORY}

A 20-year-old cob gelding presented to the University Veterinary Hospital (UVH) emergency service with a history of acute respiratory distress. The horse had been examined by the referring veterinarian three hours prior to presentation for suspected large colon impaction and was subsequently treated with an unknown quantity (estimated to be $4 \mathrm{~L}$ ) of a mixture of mineral oil and water (estimated to be $1 \mathrm{~L}$ of oil and $3 \mathrm{~L}$ of water) via nasogastric intubation. During administration of the oil and water, the horse was reported to move resulting in a brief cessation of the treatment; when treatment was resumed the horse was reported to begin to cough. Shortly after extubation, the horse began to exhibit signs of respiratory distress (increased respiratory rate and effort, profound nostril flare, anxiety). The horse was immediately treated with flunixin megluminea $(1.1 \mathrm{mg} /$ $\mathrm{kg}$, IV, once), trimethoprim potentiated sulphonamideb (24 $\mathrm{mg} / \mathrm{kg}, \mathrm{IV}$, once) and buscopan compositumc $(5 \mathrm{ml} / 100 \mathrm{~kg}$, IV, once). Two hours following the initial treatment there were no signs of improvement so the horse was referred into the UVH for further evaluation and treatment.

\section{CLINICAL FINDINGS}

On presentation, the gelding was quiet, alert and responsive, in good body condition and sweating excessively with bloody discharge from both nostrils. The mucous membranes were moist with a normal capillary refill time but slightly hyperaemic in colour. The gelding was tachycardic ( 88 beats/min) and tachypnoeic ( 26 
breaths/min), with a marked nostril flare and an increased respiratory effort with a pronounced abdominal component. Upon auscultation, there were bilateral inspiratory and expiratory musical wheezes in the caudodorsal lung fields and reduced vesicular sounds bilaterally in the ventral lung fields.

\section{FURTHER INVESTIGATION AND THERAPY}

Based on the history and physical examination, mineral oil aspiration was strongly suspected. Blood was obtained for the measurement of packed cell volume, total protein, serum electrolytes and arterial blood gas analysis. The arterial blood gas revealed a marked arterial hypoxaemia $(64 \mathrm{mmHg}$, reference range $90-115 \mathrm{mmHg}$ ) and mild oxygen desaturation (92.3\%, reference range $>95 \%$ ) while the remainder of the bloodwork was unremarkable. Based on these results, an intra-nasal cannula was placed and oxygen insufflation initiated (15L/min). A long-term catheter was also placed in the left jugular vein and treatment begun with intravenous fluids (Compound Sodium Lactated, $4 \mathrm{ml}$ / $\mathrm{kg} / \mathrm{hour}, \mathrm{IV}$ ) and flunixin meglumine (1.1 mg/kg, IV, BID). To investigate the bilateral epistaxis, resting upper airway endoscopy (unsedated) was attempted but not performed due to enhancement of the gelding's anxiety and respiratory distress. A rectal examination was performed to assess for any gastrointestinal abnormalities such as an impaction and was unremarkable. Following one hour of intra-nasal oxygen supplementation, a repeat arterial blood gas was taken which revealed a slight improvement in the arterial partial pressure of oxygen (82 $\mathrm{mmHg}$ ) and oxygen saturation (96.1 $\%)$. The gelding was maintained on the intranasal oxygen and intravenous fluids and closely monitored through the night.

The following morning, the gelding was unchanged in his demeanour and respiratory pattern, although he appeared to be less anxious and had stopped sweating. A repeat arterial blood gas revealed worsening of the arterial hypoxaemia (46.6 mmHg) and oxygen saturation (85.2\%). Blood was submitted for a complete blood count including fibrinogen and serum biochemistry. Haematology revealed a marked left shift (band neutrophils $2.11 \times 109 / \mathrm{L}$, reference range 0-0.1 x109/L), some mild toxic changes (doehle bodies) and mild hyperfibrinoginaemia $(2.56 \mathrm{~g} / \mathrm{L}$, reference range $0-2 \mathrm{~g} / \mathrm{L})$. Serum biochemistry revealed hyperglycaemia $(9.9 \mathrm{mmol} / \mathrm{L}$, reference range $3.3-6 \mathrm{mmol} / \mathrm{L})$ and hyperbilirubinaemia (75.1umol/L, reference range 5-51umol/L).

As the gelding was less distressed, unsedated resting upper airway endoscopy was successfully performed with no abnormalities detected; however, passage of the endoscope into the trachea was not attempted since the gelding became increasingly agitated during the procedure. It was decided not to re-attempt the procedure under sedation due to the potential cardio-respiratory depressant effects of the sedative agents. Ultrasonographic examination of the thorax revealed bilateral areas of ventral lung consolidation characterised by an uneven surface and numerous pronounced comet tails; there was no evidence

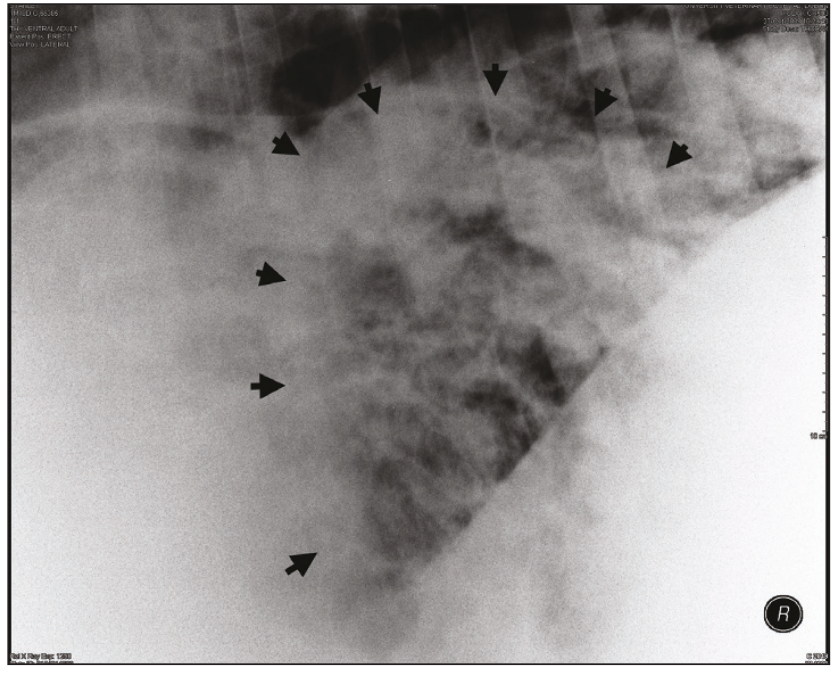

Figure 1. Lateral radiograph of the caudodorsal lung field. A mixed bronchoalveolar-interstital pattern is present (arrows).

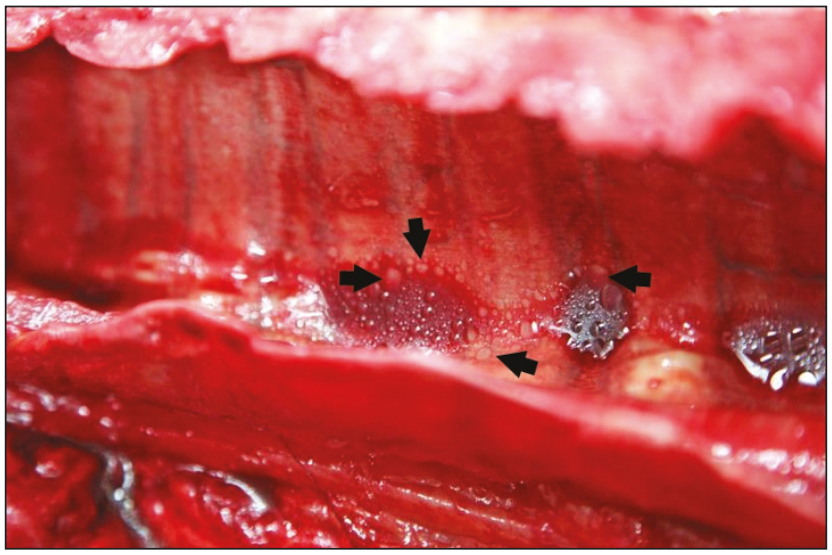

Figure 2. Gross specimen of the trachea. Lipid droplets are grossly visible within the lumen of the trachea (arrows).

of pleural effusion. A series of thoracic radiographs revealed a bilaterally severe, mixed bronchoalveolar-interstitial pattern in the cranioventral lung fields typical of aspiration pneumonia (Figure 1).

Based on the history, physical examination, diagnostic findings and continued decline in the horse's condition, an extremely poor prognosis was given and the owner elected for humane euthanasia of the gelding.

\section{POST-MORTEM FINDINGS}

Gross necropsy revealed marked congestion of the trachea, particularly in the distal half, with a large quantity of oil globlets present in the lumen (Figure 2). Pulmonary emphysema extended bilaterally over the cranioventral part of the lungs, affecting approximately $75 \%$ of the lungs. The dorsal region of the anterior lobes was severely congested (Figure 3), and a large amount of oil globules were apparent in the pulmonary parenchyma. Histopathology slides were reported to be non-diagnostic.

\section{DISCUSSION}

As with the present case, the majority of cases of lipoid pneumonia in horses present in respiratory distress with a history of nasogastric intubation (Scarratt et al., 1998). 


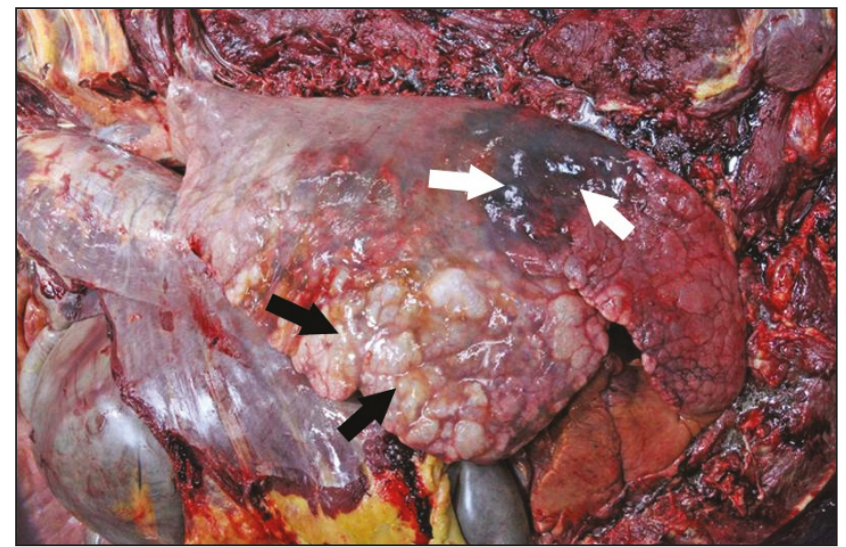

Figure 3. Gross specimen of the lung. Pulmonary emphysema is apparent on the cranio-ventral area of the lung (black arrows). Severe congestion and haemorrhage is apparent dorsally (white arrows).

However, many of the reported cases of equine lipoid pneumonia are chronic rather than acute in nature, thought to be related to the small amount of oil aspirated at the time. This is also the case in humans, with chronic lipoid pneumonia more commonly reported and thought to be due to repeated small volumes of oil aspirated over a long period of time. In comparison, the per-acute presentation of this case was considered to be due to the large volume of liquid paraffin believed to have been administered directly into the trachea as a result of dislodgement of the nasogastric tube secondary to the horse moving; to support this hypothesis, there was no post-mortem evidence of mineral oil found within the stomach or intestines.

Haematological and biochemical evaluations of horses diagnosed with lipoid pneumonia often reveal non-specific and variable changes in parameters but generally a left shift is present along with hyperfibrinogenaemia, hyperglycaemia and hyperbilirubinaemia as was the situation with the present case. In this report the fibrinogen was only mildly elevated, presumably due to the acute nature of this case while the elevation in serum glucose and bilirubin concentrations were explained by stress and anorexia, respectively. Thoracic auscultation of horses presenting with potential lipoid pneumonia typically reveals abnormal lung sounds while thoracic ultrasonography often reveals an increased number of comet tails, again supporting what was found in the present case. Early thoracic radiographic changes commonly show an alveolar and interstitial infiltrate and are diffuse, severe and non-specific (Scarratt et al., 1998, Davis et al., 2001).

Although the history and preliminary diagnostic findings may indicate aspiration of mineral oil, an accurate diagnosis is very important in establishing responsibility in these cases (Bos et al., 2002). Ante-mortem diagnosis can potentially be made by confirming the presence of lipid within the lower airway by examining fluid obtained either from a bronchoalveolar lavage (BAL) or trans-tracheal wash/aspiration (Lauque et al., 1990, Colombo and Hallberg, 1999, Scarratt et al. 2001). The presence of an oily substance floating on the surface of the fluid is thought by many clinicians to be the most sensitive antemortem indication (i.e., gold standard) of lipoid pneumonia
(Lauque et al., 1990, Sias et al., 2009). However, within human medicine there is no actual agreement on what is considered to be the ante-mortem gold standard since lipoid pneumonia is often chronic in nature resulting in an absence of lipid droplets within the BAL fluid due to the lipid being engulfed by macrophages (Lauque et al., 1990). Although lipid-laden macrophages found upon cytological examination have been used to suggest lipoid pneumonia (Scarratt et al., 2001), with the staining of macrophages with dyes specific for lipids such as Sudan B, Sudan III, Sudan IV or oil red 0 reported to further increase the diagnostic sensitivity (Lauque et al., 1990), this practice has been recently questioned (Bos et al., 2002). Furthermore, studies in humans have shown that lipid-laden macrophages in lower respiratory tract secretions do not differentiate endogenous from exogenous lipoid pneumonia but rather are nonspecific markers of parenchymal pulmonary disease such as bronchial obstruction, inhalation of organic dusts and intravenous lipid therapy (Knauer-Fischer and Ratjen, 1999). Thus, the detection of lipid-laden macrophages in lower airway secretions is not recommended as a sole means of diagnosis of lipoid pneumonia in man (Adams et al., 1997). However, studies have shown that it may be possible to distinguish exogenous from endogenous lipoid pneumonia in humans by the microscopic appearance of macrophages. In endogenous lipoid pneumonia the macrophages are frequently filled with fine, uniformly sized vacuoles with cholesterol and PAS-positive acellular debris present (Colombo and Hallberg, 1999). In comparison, large, round cytoplasmic vacuoles are seen in exogenous lipoid pneumonia (Lauque et al., 1990). Unfortunately, neither a BAL nor transtracheal wash were performed in the present case due to the level of respiratory distress the horse was exhibiting; it was felt to be inhumane to subject the horse to further invasive procedures when the prognosis was extremely poor based on the horse's lack of clinical response to treatment irrespective of further diagnostic test results.

In horses, the definitive diagnoses of lipoid pneumonia have most commonly been based on post-mortem findings of either gross demonstration of oil within the lungs or the histopathological demonstration of lipid components within affected lung tissue (Scarratt et al., 1998, Bos et al., 2002). In the current case, post-mortem examination confirmed our ante-mortem tentative diagnosis, with a large amount of lipid droplets clearly visible upon sectioning of the trachea and lung parenchymal tissue. Unfortunately, the histopathology slides were non-diagnostic due to an incorrect processing technique. Lung biopsies or sections of lung taken at necropsy from suspected cases should always be frozen to prevent removal of oil from tissues during the paraffin fixation process (Corcoran et al., 1992). This highlights the importance of providing a full history and requesting specific tests when submitting cases for necropsy. It may be necessary to identify the specific type of lipid compound in the lung tissue; chemical analysis, spectroscopy and chromatography can prove that the oil found is the same as that used for gastro-intestinal disorders (Bos et al., 2002). 
Due to the treatment history, acute onset of respiratory distress and gross evidence of oil dispersed throughout the respiratory tract on post-mortem examination, it was not deemed necessary to identify the specific lipid in the current case.

Lipoid pneumonia is much more frequently reported in the human literature in comparison to equine reports, with the most frequent causes of exogenous lipoid pneumonia being administration of lipid-based nasal drops (especially when given at bedtime) and mineral oil (as a treatment for constipation). Risk factors for exogenous lipoid pneumonia in humans include age (children are often force-fed mineral oil due to its unpleasant taste whilst in a recumbent position), swallowing dysfunction and gastro-oesophageal reflux (also a risk factor in horses). Endogenous lipoid pneumonia in humans is reported to occur secondarily to diseases that cause airway obstruction (Colombo \& Hallberg, 1999) possibly due to alterations in surfactant production in response to arterial hypoxaemia and hypercapnia (Corcoran et al., 1992). Treatment of exogenous lipoid pneumonia is similar to that reported in horses; consisting of supportive care and antibiotics if secondary complications arise. Sias et al. (2009) studied the effects of multiple therapeutic bronchoalveolar lavages for the removal of lipid-laden macrophages in affected children. Although they found considerable improvement of clinical parameters it remains to be seen whether this treatment method would be possible in horses.

It is important to note that not all cases of exogenous lipoid pneumonia in horses result from the incorrect placement of the nasogastric tube in the trachea. As with the present case, a horse may struggle during administration of mineral oil dislodging the tube from the stomach. In addition, the stomach tube may be in the oesophagus, not quite entering the stomach, resulting in regurgitation and aspiration (Stauffer, 1982). Additionally, if the stomach was abnormally distended with gas and fluids the introduction of large quantities of medication or too rapid administration can cause reflux with resulting aspiration (Stauffer, 1982). It should be noted that sick, sedated or refluxing animals may have a reduced swallowing reflex which makes it difficult to properly place the tube (Stauffer, 1982).

In conclusion, veterinarians should be aware that aspiration of fluids during nasogastric intubation is a real and serious risk and make all possible attempts to properly restrain the horse and carefully check that the tube is still in place if any movement occurs.

\section{FOOTNOTES}

aFinadyne (Intervet Schering-Plough Animal Health, Ireland)

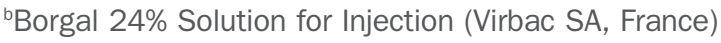

'Buscopan Compositum (Boehringer-Ingelheim, UK)

'Vetivex 11 (Dechra Veterinary Products, UK)

\section{REFERENCES}

ADAMS, R., RUFFIN, R. \& CAMPBELL, D. (1997) The value of the lipid-laden macrophage index in the assessment of aspiration pneumonia. Aust N Z J Med, 27, 550-3.
BOS, M., DE BOSSCHERE, H., DEPREZ, P., VAN LOON, G., DE VRIESE, S. R., CHRISTOPHE, A. B. \& DUCATELLE, R. (2002) Chemical identification of the (causative) lipids in a case of exogenous lipoid pneumonia in a horse. Equine Vet J, 34, 744-7.

COLOMBO, J. L. \& HALLBERG, T. K. (1999) Pulmonary aspiration and lipid-laden macrophages: in search of gold (standards). Pediatr Pulmonol, 28, 79-82.

CORCORAN, B. M., MARTIN, M., DARKE, P. G. G., ANDERSON, A., HEAD, K. W., CLUTTON, R. E., ELSE, R. W. \& FUENTES, V. L. (1992) Lipoid Pneumonia in a Rough Collie Dog. Journal of Small Animal Practice, 33, 544-548.

DAVIS, J. L., RANIREZ, S., CAMPBELL, N., \& JONES, S. L. (2001) Acute and chronic mineral oil pneumonitis in two horses. Equine Vet. Educ, 13, 230-234.

FRANQUET, T., GIMENEZ, A., BORDES, R., RODRIGUEZ-ARIAS \& J. M., CASTELLA, J. (1997) The Crazy-Paving Pattern in Exogenous Lipoid Pneumonia: CT-Pathologic Correlation. AJR, 170, 315-317.

KNAUER-FISCHER, S. \& RATJEN, F. (1999) Lipid-laden macrophages in bronchoalveolar lavage fluid as a marker for pulmonary aspiration. Pediatr Pulmonol, 27, 419-22.

LAUQUE, D., DONGAY, G., LEVADE, T., CARATERO, C. \& CARLES, P. (1990) Bronchoalveolar Lavage in Liquid Paraffin Pneumonitis. Chest, 98, 1149-1155.

LOPEZ, A. (2001). Respiratory system, thoracic cavity and pleura. In: Thomson's Special Veterinary Pathology, 3rd Edit., M. D. McGavin, W. W. Carlton \& J. F. Zachari, Eds, Mosby, Philadelphia, pp. 125-195.

NICHOLSON, A. G., WELLS, A. U., HOOPER, J., HANSELL, D. M., KELLEHER, A. \& MORGAN, C. (2002) Successful treatment of endogenous lipoid pneumonia due to Niemann-Pick Type B disease with whole-lung lavage. Am J Respir Crit Care Med, 165, 128-31.

SCARRATT, W. K., MOON, M. L., SPONENBERG, D. P. \& FELDMAN, B. (1998) Inappropriate administration of mineral oil resulting in lipoid pneumonia in three horses. Equine Vet J, 30, 85-8.

SIAS, S. M., DALTRO, P. A., MARCHIORI, E., FERREIRA, A. S., CAETANO, R. L., SILVA, C. S., MULLER, N. L., MOREIRA, J. \& QUIRICO-SANTOS, T. (2009) Clinic and radiological improvement of lipoid pneumonia with multiple bronchoalveolar lavages. Pediatr Pulmonol, 44, 309-15.

STAUFFER, B. D. (1982) Stomach intubation accidents. J Am Vet Med Assoc, 181, 448. 